\title{
Support Strategies of Certified Nurses in Diabetes Nursing for Type 1 Diabetes Patients During Puberty/Adolescence
}

\section{Ayumi Yamasaki*}

Faculty of Nursing, Osaka Medical and Pharmaceutical University, 7-6 Hatchonishimachi,Takatsuki, Osaka 569-0095, Japan

\section{Abstract}

Study objective: To elucidate the support strategy of certified nurses who provide support for type 1 diabetes patients in puberty/adolescence.

Study methods: We conducted focus group interviews with eight certified nurses who had at least one year of activity after certification and experience of providing support to type 1 diabetes patients in puberty/adolescence. The contents of the interviews were transcribed as data. We conducted qualitative and descriptive analysis with a focus on the context of conscious involvement, efforts, and thoughts toward patients being able to effectively manage their own condition.

Results: The result of the analysis led to 12 subcategories which were grouped into five main categories as follows: <<Adjusting the parent-child relationship associated with the care $>>,<<$ Linking the relationship with patients $>>,<<$ Being involved with the patient's own perspective $>>,<<$ Identifying the management method that utilizes patients' strength $>>$, and $<<$ Understanding and managing financial problems that develop from changes in the insurance system $>>$.

Conclusions: As a strategy, certified nurses interacted with patients based on the developmental characteristics of puberty and adolescence, and adjusted relationships within the family in order to promote independence of the patient. In addition, certified nurses made efforts and provided support to prevent interruption of care management due to financial problems for patients at 20 years of age, when the medical care system changes.

\section{Introduction}

The peak of onset for type 1 diabetes in Japan is 11-12 years of age; female children show a slightly higher prevalence, with just under $60 \%$ relative to male children $[1,2]$. For pediatric patients, the effectiveness of providing support at diabetes camps (held throughout Japan) specific to the developmental stages and problems which they are facing has been reported [3-5]. However, such camps are mainly focused on children up to elementary school level, and participation opportunities decrease once children reach puberty, when they have to start taking over the management of their own condition [6].

When children reach puberty, their secondary sexual characteristics begin to develop and glycemic control becomes poor, along with a dramatic increase in the secretion of growth and reproductive hormones. Sugihara (2011) [7] reported that, in the late stage of puberty, approximately $40 \%$ of patients had $\mathrm{HbA1C}$ of $\geq 9 \%$. A study that targeted females with childhood onset diabetes showed that in puberty/adolescence, they experienced deviation in the physical sensations of abnormal glucose level that they had previously acquired and, as a result, struggled with glycemic control [8]. Furthermore, as they grew, they monitored their glucose level less often and began to neglect medical care [9]. Puberty is a period during which children usually develop their identity $[10,11]$ and gender identity [12], accompanied by notable psychological changes. In adolescence, youths face decisions concerning their immediate future, such as career choices and marriage. Thus, they have no choice but to confront their own illnesses, and medical support based on these aspects become necessary.

In Japan, physicians and nurses are predominantly responsible for cooperating and providing support to patients in this age group during medical examinations. Specifically, as they are closest to patients, nurses provide medical care and support according to the patients' lifestyles. By elucidating the support strategy of diabetes and source are credited. nursing certified nurses ("certified nurses," who are assumed to have many opportunities to provide support to patients with type 1 diabetes in puberty/adolescence during outpatient visits and in hospital wards), nursing recommendations for diabetes patients in puberty/adolescence can be developed. Medical care during puberty/ adolescence may have an impact on complications that may develop during adolescence and beyond; therefore, it is considered an important period [13]. We believe that recommendations for better support for patients in this age group will contribute to the prevention of future complications; and this is the main motivation for this study.

\section{Study objectives}

The objective of this study was to elucidate the support strategy of certified nurses who provide support to patients with type 1 diabetes in puberty/adolescence.

\section{Study outline}

\section{Definition of terminologies}

Support strategy: Conscious involvement and individualized support that certified nurses provide to patients to allow them to continue managing their own illnesses with more effective practices.

"Corresponding Author: Ayumi Yamasaki, Faculty of Nursing, Osaka Medical and Pharmaceutical University, 7-6 Hatchonishimachi,Takatsuki, Osaka 569-0095, Japan, Tel: +81-72-684-7202; E-mail: fon144@osaka-med.ac.jp

Citation: Yamasaki A (2021) Support Strategies of Certified Nurses in Diabetes Nursing for Type 1 Diabetes Patients During Puberty/Adolescence. Int J Nurs Clin Pract 8: 341. doi: https://doi.org/10.15344/2394-4978/2021/341

Copyright: (c) 2021 Yamasaki. This is an open-access article distributed under the terms of the Creative Commons Attribution License, which permits unrestricted use, distribution, and reproduction in any medium, provided the original author 
Puberty/adolescence: Erik Homburger Eriksin defined the age range 12-22 years as adolescence. In the present study, we define the age range 12-22 years as puberty/adolescence.

\section{The role of certified nurses in the field of diabetes in Japan}

In 1987, the Ministry of Health, Labour and Welfare of Japan proposed the nurturing of Certified Nurse Specialists and Nurse Administrators. Following this, the Japanese Nursing Association developed a nurse certification system in 1995. Certified nurse specialists use expert nursing techniques and knowledge in specific fields of nursing, and aim to provide high-level nursing practice to those who need nursing care in various settings. The functions and roles that they are expected to fulfill include: 1) providing high-level nursing practice for individuals, families, and groups using expert nursing techniques and knowledge based on clinical thinking skills and ability to determine pathology (practice), 2) providing instruction on nursing through nursing practice (instruction), and 3) providing consultation for nurses (consultation).

To become a certified nurse specialist, one must have obtained a Japanese nursing license and have a total of at least five years of subsequent practice. Furthermore, three of the five years must be within the field of nursing to successfully receive certification. To become certified, one must complete a group education program, including at least 600 hours of practice, over a period of more than six months and less than one year at an educational facility recognized by the Japanese Nursing Association. Subsequently, one must pass a certification examination to become registered. To renew the certification every five years, nurses must complete a documented review of their nursing practice and self-development. Presently, there are certified nurse specialists in 21 different fields [14]

The educational program for certified nurses began in 2000. As of December 2019, 913 certified nurses have been registered to work in hospital wards, outpatient departments, clinics, visiting nurse stations, and educational facilities such as schools [15].

\section{Research Methods}

\section{Research participants}

Nurses with experience of providing support to type 1 diabetes patients in puberty/adolescence and at least one year of practice after obtaining certified nurse certification.

\section{Data collection methods and period}

In this qualitative and descriptive study, data were collected between February and March 2016. To select participants, we explained the objective and methods of the study to a certified nurse working at a hospital in the Kansai Region, and requested this nurse to choose appropriate candidates to participate in this research. A researcher explained the study's objective and methods, with the help of written materials, to all the nurses who were willing to participate. Eight of them gave informed consent and were chosen as participants. Data were collected by dividing the participants into two groups and conducting focus group interviews.

The focus group interview is considered to be effective in exploratory studies for expressing the different perspectives of participants, such as how they make sense of a topic, how they feel toward it, and how receptive they are toward it. It is also believed to be effective for clarifying the reasons behind people's actions [16]. In addition, this method can be used to develop new opinions as a group, through interparticipant interactions, and it does not put as much pressure on the participants as individual interviews [17]. We chose this method for our research, since our subjects had similar experiences of providing care support, and had the same educational backgrounds and roles as certified nurses.

We created two focus groups taking into consideration the participants' preferred dates and times. A semi-structured interview was conducted in a private room once with each group. During the interview, we encouraged participants to freely discuss their efforts and ideas regarding the care support they provided to type 1 diabetes patients in puberty/adolescence. We made an audio recording of the interviews after obtaining consent before preparing the data. The researcher played the role of the facilitator.

\section{Analytical Methods}

We used qualitative and descriptive methods to analyze transcripts of the recorded interviews. We extracted codes by focusing on the contexts of conscious involvement and efforts the nurses made to practice more effective care management. Subsequently, we focused on the similarities in extracted data, classified it into subcategories, then increased the level of abstraction to create broader categories. We invited researchers with sufficient experience in qualitative studies to examine the contents of our analysis and confirm the validity of our results.

\section{Ethical Considerations}

Participants were informed, both orally and in writing, about the study's objective and methods, their right to refuse participation, protection of their private information, and publication of results. Since there was a possibility of participants working together in the future at academic conferences etc., we repeatedly explained that information obtained through the interview would not be provided to any external personnel. This study was performed after obtaining the approvals of the Ethics Committee of authors' affiliated institutions (Approval number: 1333).

\section{Results}

\section{Subject background}

There were one male and seven female subjects. Their mean years of nursing experience at the time of the interview was 25.0 years (15-30 years). Their mean years of experience as diabetes nurses was 20.7 years (10-26 years). The mean number of years after acquisition of a nursing certificate was 9.0 years (1-13 years). Their affiliated departments were Outpatient (5 participants), both Outpatient and Ward (2 participants), and Nursing Education (1 participant). Their locations of work ranged among Kanto, Tokai, Kinki, and Chugoku regions.

Participants in both groups had one focus group interview each, with a mean duration of 98.0 minutes.

Care support strategy of certified nurses for care provided to type 1 diabetes patients in puberty/adolescence

Analysis of the interviews led to 12 subcategories and five categories (Table 1). 


\begin{tabular}{|l|l|}
\hline Category & Subcategory \\
\hline Adjusting the parent-child relationship associated with care & $\begin{array}{l}\text { Promoting the independence of parents } \\
\text { Being the spokesperson for the patient } \\
\text { Making the family recognize that the patient has the leading role }\end{array}$ \\
\hline Linking the relationship with patients & $\begin{array}{l}\text { Linking the relationship with normal conversation: escape from medical care } \\
\text { Dealing with the emotional expression of patients } \\
\text { Speculating the emotions behind the words and actions }\end{array}$ \\
\hline Being involved with the patient's own perspective & $\begin{array}{l}\text { Leaving the treatment responsibility to the patient } \\
\text { Minimizing the provision of support to only what is necessary } \\
\text { Waiting for the patient to develop a stance toward the illness }\end{array}$ \\
\hline $\begin{array}{l}\text { Identifying the management method that utilizes patients' strength } \\
\text { changes in the insurance system }\end{array}$ & \begin{tabular}{l} 
Identifying and utilizing patients' strength for care management \\
Table1: Care support strategy of certified nurses for care provided to type 1 diabetes patients in puberty/adolescence \\
\hline
\end{tabular}
\end{tabular}

Categories are indicated with $<<>>$. Subcategories are indicated with []. Quotes from participants are indicated with "', and supplementary text with (). Alphabetic codes in parentheses following a quotation indicates a participant's ID.

\section{$<<$ Adjusting the parent-child relationship associated with care $>>$}

This category focused on adjustments to relationships within the family, to enable the growing patient to independently practice medical care. It consisted of three subcategories: [Promoting the independence of parents], [Being the spokesperson for the patient], and [Making the family recognize that the patient has the leading role].

(I asked the mother who cannot leave her child) "What will you do when your child becomes independent (with care management)?" (G). By asking parents to imagine their child growing up, they encouraged awareness in parents and to [promote the independence of parents] toward the independence of their children.

Certified nurses were also [making the family recognize that the patient has the leading role], as shown by the following comments: "When parents try to take charge to an excessive extent, I first engage the parents and have them understand (the situation), then tackle the patient support" $(\mathrm{H})$ and "When patients reach adolescence, they are able to understand the whole treatment plan; thus, the support begins to change as the patient takes on the leading role, even if family still provides some support" (C).

In [being the spokesperson for the patient], nurses played a role in communicating with parents the patients' feelings about treatment and making adjustments to ensure that medical care continues to take place without any gaps: "A patient who had early childhood onset stopped injecting insulin themselves during the summer break. The mother did not know what to do and consulted us to see if there were any good solutions. Since it was the summer break, I suggested the mother inject the child and give the child a break. After the summer break, the patient started to inject themself again. The patient has developed a particular rhythm and a logic that he or she should be allowed to take a break from medical care during summer break. After all, the patient had always had reservations about injecting insulin." (B).

\section{$<<$ Linking the relationship with patients $>>$}

This category concerns the structure of a relationship that is prerequisite for support of patients in puberty/adolescence, who are often difficult to relate with. It consists of three subcategories: [Linking the relationship with normal conversation: escape from medical care], [Dealing with the emotional expression of patients], and [Speculating the emotions behind the words and actions].

The nurses discussed how they [link the relationship through normal conversation: escape from medical care]: "When interacting with a patient, we purposely avoid talking about diabetes, though I occasionally ask if they are injecting insulin properly. (omission) Patients know that when they come to the hospital, nurses understand that they are patients with diabetes, which they cannot talk about at school. When they talk about someone they have a crush on, we can only give ordinary answers, but we understand that they want to talk about it. So, I think it's good to talk about ordinary things. It is unpleasant if we only talk about the glucose level during an examination" (B).

Their comments also showed the importance of [speculating the emotions behind the words and actions]: "I was shocked when (a patient) casually said they want to quit insulin. They have never showed the slightest sign of such intentions at (outpatient) visits. But apparently they had been feeling like this forever. I was sorry I didn't notice." (C).

[Dealing with the emotional expression of patients] showed that the nurses, with the goal of building a relationship with their patients, had to deal with ways of emotional expressions unique to patients in puberty/adolescence: "There was a patient who seemed to want to talk but didn't say anything when I asked what was going on. (omission) But they came to the outpatient visit every month. I guess that is a phase they go through. I kept seeing this patient without quite understanding what they were going through. The patient sat facing away from the nurse as a statement. But, in truth, it was what was comfortable for them. (The patient) seemed to understand that they were here at the hospital." (B)

\section{$<<$ Being involved with the patient's own perspective $>>$}

This category is about making patients and parents become aware that the patient plays the leading role in their medical care. It consists of three subcategories: [Leaving the treatment responsibility to the patient], [Minimizing the provision of support to only what is necessary], and [Waiting for the patient to develop a stance toward the illness]. 
"It was not that (the patient) did not use insulin. But they were adjusting the amount on their own and did not regularly inject themselves. Since the patient was a university student, they had some understanding of the disease, and health care providers did not inquire about their condition. The patient will likely change once they are in the work force, but (health care providers) chose to observe the patient instead of confronting them" (H). "A patient cried during an examination saying that an interviewer for a job mistook the patient for having type 2 diabetes, yet they were unable to correct the interviewer. I wonder if the patient had learned how hard it is to be out in society. Up until now, the patient had been under the parents' protection. I encouraged them to stand up for themself regarding their disease" (G). In this manner, the certified nurses were intentionally [leaving the treatment responsibility to the patient] as a strategy of support.

In addition, the nurses kept in mind that they needed to [minimize the provision of support to only what is necessary]: "I think that many patients overcame difficulties on their own. Some patients were told hurtful things regarding work. So, I don't give advice, but offer a little bit of emotional support for aspects patients have to overcome. I think that in the end most patients overcome these difficulties on their own" (G).

[Waiting for the patient to develop a stance toward the disease] refers to intentionally giving time for patients to experience various events and change their emotions and attitudes: "It is the time in their lives where they have to find for themselves what they want to become in the future. It is when the patients make various decisions and choices. When children tell me what they want to become, where they want to go, and wanting to tackle the challenge of going out to eat with friends, it makes me think that they have grown" (B). "The glucose level in their bodies may not be at an ideal level, but children in their puberty actually have relatively decent care management. The process of building from there and allowing something to grow is important, even if the glucose level is not good" (C).

\section{$<<$ Identifying the management method that utilizes patients strength $>>$}

The nurses were [identifying and utilizing patients' strength for care management] as a strategy: "I think it would be great to utilize personality traits. University-age patients who are smart and enjoy math happily deal with CSII(Continuous Subcutaneous Insulin Infusion). If they do well, they can achieve 6\% (HbAlc)" (D) "Students of pharmaceutical science ask about duration of action when we present a new insulin formulation, just like a pharmaceutical representative. What is important is to identify and make the best out of the patient's characteristics" (D).

$<<$ Understanding and managing financial problems that develop from changes in the insurance system $>>$

This category concerns the strategy used by nurses to manage financial problems experienced at age 20 , when the health insurance system changes for Japanese people. It consists of two subcategories: [Understanding financial problems associated with medical treatment] and [Considerations and efforts toward increased share of the health care cost].

"The patient had onset during high school and was aged $>20$ when they arrived at our hospital. He began wearing CSII, but his biggest issue was money. Financial problems are something people have to think about quite a bit during adolescence and middle age. The patient was at the age where expenses for his own children became more substantial than expenses for himself" (B). "For university students and those newly at work, 10,000-20,000 yen per month is a big deal. If they run out (of insulin), they start skipping injections (since it costs money to see a doctor)" (D). In this manner, the nurses were [understanding financial problems associated with medical treatment] that arise at the age when adolescents transit into a new health insurance system.

In [considerations and efforts toward increased share of the health care cost], the nurses made efforts to minimize the patient's share of medical expenses incurred at each examination: "I carefully taught patients about tax deductions, such as travel expenses, and tried to minimize the number of glucose measurements. (When the patient is making payment) the amount that has to be paid was a little higher than expected because of a blood test. The patient was frustrated (because of the cost being higher than their budget). For this patient, the glucose level in their body is insignificant compared to whether the final bill will exceed 10,000 yen" (C). "I sometimes don't request a glucose test (insurance cover is limited). I might choose the type of insulin (based on the cost)" (B). "I reduce the frequency or postpone the examinations to make adjustments. Say, (we adjust the appointments by) changing the monthly examination to every six weeks, so we can skip one month" (F).

\section{Discussion}

We elucidated five categories for the support strategy of certified nurses who provide support to type 1 diabetes patients in puberty/ adolescence. The five categories can be further grouped into three as follows: "adjustment of the family relationship and support," "providing medical support that considers developmental and patient characteristics," and "adjustments to the environment to ensure medial care can continue."

Here we discuss two points: support strategy that includes the family, and adjustments to the environment to encourage patients in puberty/adolescence to continue with their treatment.

\section{Support strategy of the certified nurses for the family relationship}

The nurses were $<<$ adjusting the parent-child relationship while administering treatment $>>$ by [promoting the independence of parents] and [making the family recognize that the patient has the leading role] for the patient in puberty/adolescence and their family, here termed "Support necessary for the family."

Eriksin[10,11] proposed that formation and establishment of identity are developmental challenges during puberty and adolescence, which make it an important period that determines the future. For patients who had childhood onset, this is the period when they have to completely take over care management from the nurses. [6]. Under these circumstances, parents may find it difficult to determine what their own roles are and the optimum level of involvement with the patient's condition. They may involve themselves too much with trying to manage the patient's condition because they are worried. Our results showed that certified nurses asked questions of mothers who were unable to let their children become independent, so that they could objectively think about their Our results showed that certified nurses asked questions of mothers who were unable to let their children become independent, so that they could objectively 
think about their Our results showed that certified nurses asked questions of mothers who were unable to let their children become independent, so that they could objectively think about their involvement with and distance from the patient -"What will you do when they (the patient) becomes independent?"-in order to promote the independence of parents. The nurses also understood and assessed the relationship and distance between family members during conversations at outpatient examinations, and adjusted the parentchild relationship by [being the spokesperson for the patient] if the situation inhibited the independence of the patient to manage their own care.

In research that targeted patients and parents during puberty/ adolescence, it was found that patients' glycemic control was good if the parents had a democratic attitude to their children, and if there was agreement in their communication style [18]. If parents were more controlling, patients had poor control [19]. These previous studies show that excessive interference by parents likely inhibits the independence of patients.

It has been shown that mothers of chronic disease patients in puberty/adolescence experienced conflicting emotions about accepting their child's disease and remaining positive, while being anxious about changes in their child from living with an unpredictable disease [20]. The nurses were [promoting the independence of parents] and [making the family recognize that the patient has the leading role] by not criticizing the parents for being excessively involved, but instead intentionally helping the parents to realize that their child must play the leading role.

In Japan, $29.5 \%$ of unmarried people aged 20-34 years were living with their parents in 1980; by 2016, this proportion had increased to $45.8 \%$ [21], for various reasons, including the financial ones. Compared with young people not living with their parents, the mutual distance is likely closer. Puberty/adolescence is a period of moving on to the next level of education or starting employment. For patients, it is a period of establishing independence in society and in their own medical care. However, when living with their children, parents may be show more interest than necessary in their lifestyle and care management. The nurses considered these realities that Japanese families find themselves in and did not dismiss the parents' concerns, instead becoming consciously involved to make efforts to prevent interference in the patients' independence of their own medical care.

\section{Strategies to promote continuation of the treatment while considering the period of puberty and adolescence}

The nurses [understood financial problems associated with medical treatment] and gave [considerations and efforts toward increased share of the medical expenses]. In this manner, they were $<<$ understanding and making efforts to manage financial problems that arise when the social welfare system changes $>>$. They provided support to prevent interruption of treatment due to financial problems.

In terms of treatment of pediatric chronic diseases, Measures against specific pediatric chronic diseases were formulated as a project based on the Child Welfare Act. It provides public subsidy for the patient's share of medical insurance for patients aged $0-20$ years [22]. Type 1 diabetes is included in this measure, but as soon as patients turn 20, they are no longer eligible for the public subsidy, regardless of their employment situation, and are returned to the general medical insurance system. To manage type 1 diabetes, in addition to a monthly outpatient examination, one requires tests such as blood tests and urine analysis, insulin and needles for injection, devices to self-monitor glucose level, and disinfectant wipes. While it may vary for each patient, the treatment expenses can amount to tens of thousands yen per month.

It has been reported that, for both type 1 and type 2 diabetes, self-monitoring of glucose level is more useful for glycemic control when the number of measurements is high [23]. However, the level of such monitoring is limited by the insurance system. Therefore, [considerations and efforts toward increased share of the medical expenses], i.e., "carefully considering tax deductions, including travel expenses, and minimizing the number of glucose level measurements" is an important and characteristic strategy for patients in this age group.

For patients older than 20 years who are no longer eligible for public subsidy, there exists a "high-cost medical expense benefit" system which reimburses medical treatment costs if they exceed a certain threshold. In a study that examined patients who turned 20 after having previously received the public subsidy for specific pediatric chronic diseases, however, it was shown that 59\% of these patients did not receive any subsidy for medical expenses [24]. In addition, patients with childhood onset type 1 diabetes were not well informed about their medical expenses [25]. It was assumed that the family, (especially parents) who share a livelihood paid the medical expenses for examinations. When these patients passed the age of 20 years, they become aware of the actual expenses associated with treatment of their disease which they were previously unaware of due to the public subsidy. This became a major burden for some patients.

Patients in this age group become aware of themselves in society, begin social activities, and gain an outlook for their own livelihood and future [26]. However, there are individual differences in this developmental process, and some patients struggle to verbalize their problems. What is important for the certified nurses involved during an outpatient examination with adolescent and early-adulthood diabetic patients who have difficulty finding support is to continuously stay involved, understand what is not said, but not get too closely involved [27]. The care support strategy of the certified nurses we interviewed was: <<linking the relationship with patients $>>$ by [linking the relationship through normal conversation: escape from medical care] and [speculating the emotions behind the words and actions] and <<being involved with the patient's own perspective $>>$ by [keeping the support to what is necessary] and [waiting for the patient to develop an outlook toward the disease: trusting the patients and waiting]. Such support leads to psychological support and trusting relationships, prevents interruption to medical treatment, and manages financial problems.

\section{Conclusion}

In the strategy of the certified nurses who provided support to type 1 diabetes patients in puberty/adolescence, five categories were identified: $<<$ Adjusting the parent-child relationship associated with the care $>>,<<$ Linking the relationship with patients $>>$, $<<$ Being involved with the patient's own perspective $>>$, $<<$ Identifying the management method that utilizes patients' strength $>>$, and $<<$ Understanding and managing financial problems that develop from changes in the insurance system $>>$. 
We divided these categories of support strategy into: "adjustment of the family relationship and support", "support that considers developmental and patient characteristics", and "adjustments to the environment to continue care". It was shown that the certified nurses' strategy was not only to provide support for the patient and family, based on developmental characteristics, but also to respond to financial problems that arise for those in the age group when the medical care system changes.

\section{Acknowledgements}

We would like to extend our most sincere appreciation to all participants who cooperated in the present study.

\section{Funding}

The study was funded by Grant-in-Aid for Scientific Research of the Japan Society for the Promotion of Science.

\section{Competing Interest}

The authors declare that they have no competing interests.

\section{References}

1. Amemiya S (2011) Characteristics of Japanese people with childhood and adolescent diabetes and future challenges. The Journal of the Japan Pediatric Society 115: 13-19.

2. The Japan Diabetes Society, The Japanese Society for Pediatric Endocrinology (Eds.) (2011) Pediatric and adolescent diabetes management handbook, Revised 3rd Edition: Consensus guidelines, Tokyo, Nankohdoh. pp. 50-65.

3. Yakusijin Y, Nakamura, K Okada T, Takeda A (2012) Educational benefits of a diabetes camp for children with type 1 diabetes. Journal of the Japan Diabetes Society 55: 866-873.

4. Nakamura K (2005) Support for children with type 1 diabetes and their families. Journal of Japan Academy of Diabetes Education and Nursing 9: 37-43.

5. Nakamurai K, Ito T, Umedai E, Ohnoi T, Kida K (1998) Effect of international diabetes camp for Japanese participants. Journal of Japan Academy of Diabetes Education and Nursing 2: 97-103.

6. Maeda, H. Soeda, K. (2013) Transition of care behavior from parents to children in case of children with type 1 diabetes as observed from the parent-child relationship. Journal of Japanese Society of Child Health Nursing 22: 9-16.

7. Sugihara S (2011) From a Diabetes Perspective. The Journal of Child Health 70: 41-45.

8. Yamasaki A, Tomari Y, Takaya R, Ishiro M (2016) The Process by Which girls who Develop Type1 Diabetes Before School Age Acquire Self-Management Skills During Puberty and Adolescence. Health 8: 1788-1806.

9. Yamasaki A (2018) Process of Becoming Capable of Self-Management during Puberty/Young Adulthood in Boys with Type 1 Diabetes Mellitus Beginning Prior to Elementary School. The 6th Asia Pacific Congress of Pediatric Nursing 74.

10. Erikson EH, Erikson JM (2001) The Life Cycle Completed: Extended Version, (T. Murase and K. Kondoh, Trans.), Tokyo, Misuzu Shoboh.

11. Erikson E.H (1950) Childhood and Society, Vol. 1 and 2. (Y. Nishina, Trans.) Tokyo, Misuzu Shoboh.

12. Matsumoto K (Ed.) (2006) Sex: the nursing of sexuality - aimed at realizing QOL [In Japanese]. Kenpakusha, Tokyo, pp. 63-64.

13. Kuniyoshi M, Gushiken M, Miyagi K, Gajya S (2003) Self-care behavior of diabetic children and its practice in school life. The Journal of Japan Academy of Diabetes Education and Nursing 7: 107-114.

14. Japanese Nursing Association, about certified nurses.

15. Japanese Nursing Association, certified nurses based on data: The list of registrants by fields and prefectures.
16. Focus group interviews in education and psychology, Osamu Inoshita, supervisory translator, Jun Tabei, translator. Keio University Press Inc, Tokyo.

17. Anme T (2001) Group interview method in human services: development of qualitative study based on scientific evidence. Ishiyaku Publishers, Inc. Tokyo.

18. Chien Shu Chun (2002) A study on self-care and perceived relationship by type1 diabetic adolescents and their parents in Taiwan. Journal of Chiba Academy of Nursing Science 8: 1-8.

19. Kawaguchi T, Maruyama H, Kawata C (1997) Family environment and diabetes control in IDDM adolescents. Journal of Japan Academy of Diabetes Education and Nursing 2: 97-103.

20. Sasaki M, Kojima $H$ (2017) Thoughts of mothers about the independence support for junior and senior high school students with pediatric chronic disease. Journal of Japanese Society of Child Health Nursing 26: 1-7.

21. Nishi F (2017) Current Situation of Unmarried Persons Living with Their Parents: Statistical Research and Training Institute, Ministry of Internal Affairs and Communications.

22. Information center for specific pediatric chronic diseases, Japan: regarding medical subsidy for specific pediatric chronic diseases.

23. Nishimura A, Wasa M, Harashima S, Nin K, Inagaki N (2018) Influence of flash glucose monitoring on diabetes self-management: a before-after study. Journal of the Japan Diabetes Society 61: 171-180.

24. The health science and labor sciences research grants, a basic study project on the growth of next generation such as overcoming diseases, a study on situations and needs of carryover patients of specific pediatric chronic diseases, 2011 study report, supervisor, Toshiyuki Ojima, pp. 5.

25. Sakamoto $T$ (2015) The reality of social independence and control of type 1 diabetic patients. Journal of the Japan Innovative Diabetes Treatment 11:11-15.

26. Nagata H, Psychology for Nursing Students. IGAKU-SHOIN Ltd. Tokyo. pp. 79.

27. Tsuda H, Noguchi E, Shimizu Y (2018) Outpatient nursing for adolescents and young adults with diabetes where it is difficult for nurses to find opportunities for support, Osaka University Knowledge Archive 24: 35-43. 